Journal of Social Sciences 1 (4): 203-210, 2005

ISSN 1549-3652

(C) 2005 Science Publications

\title{
The Role of Information Technology in Building up Knowledge Economy Producting and using Information in Libraries and Information Centers
}

\author{
${ }^{1}$ Dr.Ibrahem Mohssin and ${ }^{2}$ NedaL AL-Ahmad \\ ${ }^{1}$ Library and Information Science, Zarka Private University, Zarka, Jordan \\ ${ }^{2}$ Library and Information Science, Al-Balqa Applied University, Prince Aliah College, Amman, Jordan
}

\begin{abstract}
The study reveals the relation between information production processes and building up knowledge economy and clarifies the role of libraries and Information Technology in producing information for the purpose of using it in bulking up knowledge economy.
\end{abstract}

Key words: Information technology, producting information, knowledge economy, information society, knowledge society, library, information centers

\section{INTRODUCTION}

The significance of the study: The significance of the study lies in exploring the correlation between Information production processes, building up knowledge economy and demonstrating the importance of libraries and Information Technology in Information production and using it to build a serious knowledge economy.

The previous studies: Under the title of "The role of university libraries in developing scientific research and satisfying the needs of researchers", Mijbil Lazem AlMalki touched upon the nature of scientific research and Information production, research patters and role in development, as well as the correlation between university libraries, scientific research, Information production methods, researchers' need for Information and the role of library in this (Resalat Al-Maktba, vol. 38 ,No3,4). Yakov Shraiberg presented a study in the twelfth international conference for "Library and Information Resources in the Modern World of Science, Culture, Education and Business" under the title of " Library and Universities in the Digital Information Environment: First Step on the way from Information Society to the Knowledge Society" in which he discussed :the main future of the library, information and educational at the current stage of the human society evolution this stage in characterized by the transition from the information society to the society of knowledge which is the final goal of social transition in the information age, analyzed are the main key stone of the transition i.e computer and communication technology status, legl support libraries and universities in providing information and education, etc (http:// www.gpntb.ru (CD-ROM available )). In a study which was presented in 9th International Conference (LIBCOM 2005), that was held by International Library and Information Analytical
Center, Mohssin, Ibrahem discussed the relation between Information Technology and libraries, Information Technology and scientific research, as well as scientific research and libraries. The research arrived at the following results: discussed the correlation between Information Technology and libraries, Information Technology and scientific research, as well as scientific research and libraries. The researcher analyzed the concepts of library, information, scientific research, science, knowledge and Information Technology. In addition to that, the researcher found a correlation between libraries, scientific research and Information Technology and identified the role of Information Technology in libraries and scientific research. One of the results the researcher arrived at was that libraries, scientific research and Information Technology are inseparable (http:// www.iliac.org).

Helmi Abu Al-Futooh and Abdellbaqi Abu Zaid discussed in their study entitled "Communication Technology and its Educational and Social Impacts: a field study in the kingdom of Bahrain" the impact of Information Technology on education quality, social relationships and the future of societies (http:// www.khavma.com/education-technology/s20htm). In the preparations of the United Nations Economic and Social Commission for Western Asia (ESCWA) for the World Summit on the Information Society 2002, there were three studies under the titles "Some selected issues in the field of Information Technology and communications given priority by the ESCWA", "The infrastructure of Information Technology and communications in the countries of ESCWA" and "The coordination of Information Technology and communications standards related to using the Arabic language in the Information society". The first study deals with the impact of Information Technology and communications on social and economic development in the countries of ESCWA, the challenges and the pivotal issues that encounter the region, as well as the

Corresponding Author: Dr. NedaL AL-Ahmad, Professor, Assistant in Library and Information Science, Zarka Private University-Jordan, Tel: 962-777-428415 
current polices of this region. The second study describes the status of the infrastructure of Information Technology and communications in the region, analyzes the achievements and recommends developing the infrastructure in order to meet the new requirements of information societies. The third study highlights the major problems that hinder the extensive use of the Arabic language on the internet and recommends the adoption of a unified regional policy for using the information provided by the internet (http://www.escwa.org.lb/arabic/division/sociaty.html).

The purpose of the study: The study aims at illustrating the correlation between Information Technology, libraries and Information production in order to build up knowledge economy.

The methodology of the study: The researcher has followed the pattern of studying the relation between variables, identifying the variables in Information Technology, Information production, knowledge economy and library, as well as displaying the correlation between these variables and the role played by each one in enforcing the role of other variables on the basis of benefit integration.

\section{The problems of the study}

* Studying Information and Information Technology and their impact on the processes of handling and processing Information.

* Studying the role of Information Technology in building up knowledge economy through producing Information in its different shapes and patterns.

* Studying the role of libraries in building up a serious knowledge economy.

* Studying the patterns of Information production in knowledge economy.

First subject: Information technology and information development: The term Information Technology is made up from two parts Information and Technology which prompt us to study the two terms. Technology is derived from the Greek word "TECHNE" which literally means an art or craft but as a concept it means all the methods and tools dedicated for helping in executing the productive and unproductive processes. The common conception for Technology is that it is the process of total or partial substitution of the human productive functions with automatic methods for the purpose of facilitating the work and raising the level of production. It should be mentioned here that Technolgy is used most of the time to refer back to and describe modernism. Information on the other hand is derived from the Latin word "INFORMATIO" which literally means classifying and summarizing. Information is considered to be a tool dedicated for delivering knowledge and means the row data that can be exchanged between people through the oral or written methods. This term is used most of the time with the processes related to computer ${ }^{[1]}$.

Information is essential for building up knowledge economy and developing the economic, political, social and cultural resources, for some people believe that the elements of production are the materials, power and Information.

The term technology includes biological, agricultural, transportation, medical, military, art, education, media, printing technology and others. Education, media and printing technology come close if not blinding sometimes with Information and library technology. Therefore, Information Technology is considered to be inseparable part of technology in general and library technology is inseparable part of Information Technology, for instance a technology like microfilms which is almost limited to libraries and Information and documentation centers is fundamentally part of Information and education technology. So, we can say that Information Technology is computers, methods and tools that are used to automatize and mechanize the process of searching in Information. Put it another way we can say the Information Technology is the technical methods that are dedicated to: collecting, recording (storing and saving), processing, spreading, delivering, as well as producing Information. By reviewing the concept of Information Technology we realize that computer is not just a part of Information Technology, but the most important part of it.

We realize from what have been mentioned that Information Technology facilitates the process of collecting, recording, processing, delivering, producing and spreading Information. These processes participate in accomplishing Information production processes, managing and organizing libraries and Information centers.

Information production processes, building up knowledge economy and reinforcing Information societies have gone through the following stages:

First: Collecting information: Scientists estimate that people obtain $75 \%$ of Information through vision, $13 \%$ through the sense of hearing, $6 \%$ through the sense of touch and 3\% through the sense of smell and that human brain uses only $1 / 7$ of its comprehension capacities. Therefore, people have sought in the course of time to develop methods for collecting and storing Information since they wrote this Information on walls until they were urged by necessity to create Information Technology capable of storing unlimited forms and sizes of Information resources ${ }^{[2]}$.

Second: Recording, storing and processing Information: In order for Information to be useful it should be stored and delivered to beneficiaries as quick as possible. 
The processes of recording, storing, processing and reshaping Information have gone through the following phases:

The phase of writing emergence: This phase included the appearance of picture and alphabet writing, font development and the invention of printing press. This phase also witnessed inscribing writings, 5000 years ago, on walls, clay tablets, animal skins, papyri and other materials.

The phase of inventing paper: It is well known that Chinese are the first to invent paper. The original forms of books at that time were in shape of rolls and were called "manuscripts".

The phase of inventing movable letters: Chinese invented movable letters which led to the invention of printing and helped in duplicating books in large numbers which enabled people to store various copies and distribute them in different places.

The phase of inventing computers and other Information Technology that help in storing Information: In this period many technologies that help in recording and storing have appeared such as: microfilms, audio and video tapes, audio-visual tapes and magnetic disks. This period is still full of developments that we are witnessing nowadays and will be witnessed more extensively in the upcoming years. At first, people have relied on storing Information in their minds but they faced the problem of forgetting which prompted them to find more efficient methods. Therefore, people developed libraries and documentation centers and gave libraries greater attention until they became the most spread centers for storing Information. People have also developed different equipment and technologies and have created expert systems which form artificial intelligence and transform machines into expert workers in one or more fields. In addition to that, people have developed different methods for storing Information which are small in size but big in capacity and can be used to retrieve Information a more accurate and quick manner. All what have been mentioned and much more have enormously participated in the process of storing Information $^{[3-5]}$.

The fundamentals of storing and retrieving Information are based on providing Information Technology capable of establishing and updating data bases, inputting data (storing Information), retrieving and presenting Information, providing confidentiality and Information security and providing the fundamentals of searching for and retrieving Information.

Third: delivering and spreading Information: Before the appearance of writing the main method for
Information exchange was the conversation between people who have been facing the problem of total or partial oblivion in addition to twisting (over or under statements). So people have sought to create new methods to help them not to forget and avoid twisting facts by creating writing which participated significantly in facilitating the process of spreading Information. Yet, writing encountered the problem of the diversity in font patterns and styles which led to the appearance of different copies of the book. In addition to that, hand writing process used at that time was slow and needed lots of time and effort and that is why man invented printing press which helped in solving the problem of the diversity in font patterns and styles and consequently solving the problem of the different patterns of copies. This process participated in a way or another in delivering books to different distant places by using the services provided by mail, for mail has been the most used method of communication. Postal system in turn used different types of transportations (ships, trains, planes...etc) to deliver books. Electricity has played an important role in spreading Information through operating phones, telegraphs, fax machines and others which have played a significant role in transmitting Information from one place to another round the world. Radio and television have also played an important role in transmitting audio, visual, or audiovisual Information through technologies such as Teletext and Videotext. The massive developments in satellites technology led to the appearance of specialized educational-Information al channels, some are paid and some are free (like Arabsat and Nilesat educational channels) ${ }^{[6]}$.

Communication and Information revolution (the association between Communication and Information). This period has witnessed a broad spread of communication and tremendous developments in the technologies of computers and communication which led to the appearance of general and specialized networks as well as merging many of these networks which ultimately led to the emergence of the internet which participated in spreading Information electronically.

Fourth: Information production: The importance of Information Technology for building up knowledge economy lies in this point, since the main components of Information production and duplication which are: writing, editing, translation, advertisements, meetings and others are considered to be the bases of every decision taken by any director in his field. Decision making depends on: the quality of Information related to the problem under discussion, the validity of the Information, as well as the current need for Information and that is why people are motivated to collect, store and organize Information for the purpose of retrieving it as quick as possible and with minimal effort. This represents the process of Information production for the decision maker ${ }^{[4,5]}$. 
There are basic foundations for building up knowledge economy, the most important of these are: the researcher, the laboratory and the Information center, LIBRARY, or documentation center and others. This point clarifies the role of Information in the process of transforming Information and rearranging thoughts in order to reach for new Information (Information production), discoveries and inventions. Therefore, we cannot find a good researcher who does not make use of Library or Information centers ${ }^{[3,5]}$.

The role played by Information Technology in Information production processes is restricted to: First, Participating, partially or totally, in Information production. For example, most corporations produce Information with the help of Information Technology. This production is represented mainly in Information about income, purchases, expenses, wages, salaries and others. This Information is produced with the help of specialized Information Technology represented in software and hardware. Second, organizing and facilitating the process of retrieving Information resources, like data on rival corporations, general statistics, laws, regulations and others. This Information is also produced with the help of specialized Information Technology represented in software and hardware.

Second Subject: The role of information technology in producting different types of information till building up knowledge economy: The first official admission with the problem of how to deal with the rapidly changing world in the twentieth century and afterwards was in 1945. After the admission of this problem it was normal that intellectuals will have to face this problem. This was done through the attempts of library specialists and the engineers who are concerned with organizing knowledge, so as to create new methods for storing and retrieving Information in order to provide Information services. Those scientists have helped in applying new methods for preparing data and using these data in libraries and documentation centers through using Information Technology, especially computers.

In order to understand the relation between Information science, knowledge economy and Information production, it is necessary to identify the fundamentals of this science which are: knowledge, which is demonstrating the issues and the relation between them, researching and publishing, the nature of Information production and different Information resources, acquiring and organizing knowledge, the methods of acquiring and organizing Information resources through classification and cataloguing, storing and retrieving knowledge, as well as the methods of presenting Information, planning, management, system analysis and Information Technology especially computers ${ }^{[7,8]}$.
Therefore, the process of producing Information with its different methods, forms, am patterns benefit from Information Technology, this is because it benefits from the different forms of Information and analyzing them in order to conclude from.

Information that produces and builds up knowledge economy lies within three patterns of Information production ${ }^{[9-12]}$ :

* First: the new Information produced through describing phenomena without changing the available data.

* Second: the new Information produced by studying the events that took place in the past.

* Third: the new Information produced by deliberately changing the available data in order to affect it (changing it)

The new Information produced through describing phenomena without changing the available data: which is Information that describes phenomena and objects through: observing the phenomena, collecting facts about these phenomena, specifying the special conditions of these phenomena, explaining them and then determining the situation through describing the reality as it is. This process includes a set of forms which extensively make use of Information Technology like: producing Information by scanning the available reality, producing the Information which studies the correlation between two phenomena through studying each one individually and explaining the effect of one of them on the other. Producing Information which is based on studying the relation between different variables may exceed describing the variables and the relation between them to explain the reasons for this relation. other forms of the Information produced by describing phenomena branch out of producing Information that studies the correlation between two or more phenomena which are called: "the pattern of producing Information that explains the reasons of the relation between different variables" and "the pattern of producing Information that studies the variables resulted from time passing on the application of studies and researches. This pattern depends on producing new Information on the efficiency of applying a study or a research in reality by going back to the results of these studies after a certain period of time from their application. Finally, we have the pattern of the new Information that collects data about a certain case (describing a certain case) and tries to apply the special features of this case over a group of closely related cases. For example, the producer of new Information by describing phenomena without changing the available data makes use of Information Technology in executing statistical operations representing them easily in graphs; all he needs is a simple knowledge in statistical applications whereas this operation used to require lots of work, effort and accuracy. Therefore, computer 
technology provides, through special software, effective applications to transform data into statistical Information or graphics through inputting the data and clicking one or more buttons to execute the operation and finally getting the statistical Information on paper. In addition to that, Information producer benefits enormously from library items like Information recourses and that is through the facilities provided by the different Information technologies especially the computer technology and its different programs(Access, excel, M2L ....)

The new Information produced by studying the events that took place in the past. It is the process of studying the past events that occurred in the past depending on vestiges, records, newspapers, magazines, eyewitnesses, memoranda, autobiographies, previous studies, literary works and others and benefiting from these at the present time and in predicting future. For example, one of the most important Information recourses in the historic researches is manuscripts. Here, Information Technology helps in providing these manuscripts in the shape of microfilms magnetic tapes, or CDs as well as helping in organizing the library items using different Information Technology methods. On the other hand, the new Information produced by deliberately changing the available data in order to affect it (changing it) is the new Information that is based on making changes in the requirements of the current situation through entering variables that change to change this situation, in a way that restrain all variables except for the one we want to study its effect. This process aims at studying this variable in the light of the changes that occurred in the given data.

In this field, Information Technology helps in preparing and designing the tools which help the researcher in collecting Information about the variables that are subject to be studied in the research, like questionnaires, tests, the different types of interviews, as well as helping him in sorting the results extracted by these tools and identifying the statistical and descriptive data.

The factors that have led to Information explosion and subsequently seeking to create the Information that is capable of satisfying the current requirements of Information explosion and knowledge economy are: the growing increase of discoveries and inventions, the overlap of different branches of knowledge and the emergence of new specializations (the appearance of what is called major and subsidiary major). In this century, people have acquired Information that exceeds what they have acquired in the course of their long history.

Third subject: The importance of using information in libraries to build up knowledge economy and knowledge society: The importance of using Information Technology in Libraries, Information centers, researches and studies lies in solving the problem of the tremendous increase in the human intellectual production and the problem of the new demands on Information. The rapid scientific development, the overlap of the scientific majors and the appearance of new subsidiary majors pushed towards focusing on the Information itself rather than its resource, i.e. turning away from possessing books and other resources of Information into just providing Information and moving from general Information resources into specialized ones and the appearance of what is called the general and specialized Information banks. All this led to the change of the nature of Information resources and consequently the appearance of specialized LIBRARIES and Information centers that are concerned in organizing researches and studies that improve the outcomes of specific institutions ${ }^{[8]}$.

We can say here in derails that Information Technology is used in libraries, Information centers and Information production works to build up a serious knowledge economy through:

First: Using information technology in the question process: Information Technology helps in making lists of the library needs, perusing and completing the procedures of ordering these items, delivering them, asking for the delayed items, identifying the bibliographical data related to the library item to facilitate purchasing procedures, presenting statistical data and financial Information. In addition to that, Information Technology helps in the processes of creating primary records for the materials entered in the library which are then modified in the indexing and classification sections. Information Technology with its great benefit on the question process leads to an enormous benefit on the processes of producing new Information, for if the basis of producing Information is the Information itself, then the process of supplying this Information will surely benefit Information producers. Information Technology organizes the processes related to the providing and developing the library collections in a more accurate, quick and productive way than the traditional way.

Second: Using information technology in the process of controlling periodicals: Periodicals need a special Information Technology treatment, since bibliographical description of magazines and other periodical Information resources differ from books and that requires a special treatment. Information Technology also helps in specifying the dates of the periodicals arrival, specifying the delayed editions for the purpose of claiming them, determining the periodicals that have been cancelled or have changed their titles, issuing a list of the periodicals which the library is subscribed to and specifying the dates of renewing subscriptions, as well as helping in issuing statistical reports about periodicals. Some periodicals are available as an Information resource in the shape of 
microfilm, microfiche or different types of disks. Here we should not forget that periodicals are one of the most important Information resources if not the most important for the Information producer, since they include the most recent Information related to his research.

Third: Using information technology in cataloguing and classification: Information Technology helps in the process of cataloguing and classification through electronic catalogues. They provide the ability of modifying the records that have been previously entered, adding new bibliographical records, omitting the bibliographical records are already there, Printing out catalogue cards, issuing lists of the new additions, making use of the congress library records and records from other libraries which are in the shape of microfiche (automatized cataloguing)and also available as (CD-ROM). So, the process of organizing Information resources available in the library is based on cataloguing, classification and organization which mean that if library collections are organized properly according to accurate bases in cataloguing, classification and indexing, the mistakes in the process of organization will be reduced and the accuracy in putting library items on the right library shelves will be increased. So, if an advanced technology is used in such processes, then Information procedures will obtain, which is the basic part of Information production processes and subsequently building up knowledge economy in highly accurate and fast manner ${ }^{[1,7]}$.

Fourth: Using information technology in public services sections: Public services sections mainly include four major parts: the first one is the lending section which provides the Information producers and libraries with: the help in completing the processes of lending the library items and retrieving them, the help in the mutual lending between different libraries in the country, i.e. sharing the usefulness of library items.

The second one is the referential services which are provided by Information Technology for Information producers and libraries like: helping in answering the questions of the readers and in accessing the resources through the processes of cataloguing, classification and organization based on Information Technology and providing some resources like dictionaries and encyclopedias in the shapes of microfilms or compact disks (CD-ROM) or through the on-line method. The third is (On Line Public Access Catalogue (OPAC)) which helps Information producers and libraries in: decreasing the crowds over the traditional cards catalogue through providing the library or the mother institution departments with computers, facilitating the process of accessing Information resources by using entries like: the author, the title, the publisher, or others. On the other hand, the process of mixing entries can be done by using part of the writer name or the title or using a mixture of both. The fourth one is the service of compact disks (CD-ROM) and making use of different types of data bases ( dictionaries, encyclopedias, ...etc) which are provided to Information producers and libraries in the shape of (CD-ROMs). In addition to that, some publishers provide libraries with lists of their new publications in the shape of (CD-ROMs) or publishing them directly on the internet. All these services and much more depend basically on the type of the Information Technology used ${ }^{[5,13,14]}$.

Fourth subject: internet and its rule in information industry and building up knowledge economy: Nowadays, internet serves as a connective device between the Information user or producer and different data bases. Internet is a group of computers distributed all over the world, put it other way, it is a group of data bases that merge to form Information banks which in turn merge to form an Information net which if merged form what is called "The internet". The internet can help in the library works, in the process of producing Information, preparing theses and researches and writing processes and others. Here, we can consider the internet as a vast group of libraries merged together, or merged with Information centers and banks and documentation centers. Then we can think of the internet as a big unlimited source of Information. The fields in which the Information producer can make use of the internet are: exchanging experiences and opinions between scientists and specialists in the same field around the world and learning about the latest developments in their researches through exchanging the Information using the Electronic Mail (e-mail). The internet provides the ability to: access Information from more than one research center around the world, establish associations in specific fields of knowledge or specific specializations and provide the possibility to make use of the Information resources that exist in libraries in most of the world countries. This cooperation between libraries is represented in the electronic lending and supplying, serials control, accessing what is called the electronic Catalogue (ONLine Public Access Catalogue (OPAC)), as well as exchanging software, pictures, recordings, and others through a special protocol provided by the net ( File Transfer Protocol (FTP)) and examining the library's Catalogues ${ }^{[15]}$.

The net also provides referential services like: providing the necessary methods to benefit from the available Information resources, answering the readers and Information producers' inquiries about certain subjects by providing bibliographical or statistical data, abstracts or complete texts, as well as providing Information producers with certain forms of current services. The net also provides electronic and digital texts and offers exploring library items for those who want to examine its Information resources and benefit from them for a subscription paid when asked for. It 
should be mentioned here that most of the libraries catalogues in the developed countries (the countries of west Europe and North America) provides these services in the internet and also provides some on-line referential services through the internet which are represented in the Information inquiry system through the on-line access to Information sources like encyclopedias, indexes, catalogues, dictionaries and other Information resources which help obtaining media Information. One of the advantages of the internet is that it provides its users with a level of freedom in producing spreading and benefiting from Information. The internet breaks the barriers between the Information producers and develops their abilities of communication and consequently makes it possible to participate in and share opinions. It also provides a new method for searching in Information depending on acquiring the Information in its two sides, the Information al and the educational. It also eliminates the geographical boundaries between countries and the places where the Information producers exist. The services presented by the internet for the users and library staff lies in: acquiring Information resources and benefiting from external Information resources like the e-mail which can be considered as a personal post office from which we can send and receive messages and attachments. In addition to what have been mentioned, e-mail is better than the ordinary mail in that it works 24 hours a day and receives messages even if the computer is shut down. The e-mail provides the possibility to answer the questions of Information producers in a high speed and to inquire about new publications from the publishers, the dealers, the libraries and others. In addition to what have been mentioned, the internet provides file transfer service which works according to (File Transfer Protocol (FTP)) which helps in exchanging Information between experts (specialized Information producers) in one or more related fields. The internet also provides the TELNET service which facilitates the process of communicating and searching for Information through entering another computer from yours and operating it as if the keyboard of that computer is in front of you. To access this service, the Information producer must know how to deal with the computer he accessed and to be allowed by the other computer to enter its files or break into its security programs. This technology helps Information producers in contributing to researches without changing the place of residence and work. The internet also provides Information service, which is presented by the Information server for vast areas. This technology helps in looking for texts in a vast group of data bases, in addition to the ability of choosing the data base or obtaining the Information without determining its place (on which net or web site). It is also possible to access the Information on the net by moving through the hyperlinks or hypertexts ${ }^{[14]}$.
Therefore, who possesses the Information possesses the world and rules the universe and also possesses the ability to produce Information, i.e. the ability of searching for the purpose of producing new Information which in turn can be transformed into inventions or discoveries hence, building a serious knowledge economy.

\section{RESULTS}

There is a strong relation between Information Technology, libraries and Information production.

Information production plays an important role in building knowledge economy.

There is a strong relation between the different types and patterns of Information production.

\section{RECOMMENDATIONS}

Implanting the different forms and patterns of knowledge production through talking about them more often in media and organizing seminars, lectures and workshops to clarify and show the importance of each one of them. It is also necessary to include them as a course in the different academic stages whether in schools and universities.

Using the correlation between Information Technology and knowledge production processes in one hand and the potentials of Information Technology in libraries for building up a more efficient knowledge economy.

Improving the abilities of those who are responsible for Information production in dealing with Information Technology through the continuous processes of teaching and learning.

Establishing centers specialized in producing the Information that is important to develop knowledge economy under the supervision of institutions responsible for controlling and permitting the spread of such Information.

\section{REFERENCES}

1. Moodgel, H.M.K., 2001. CD-ROM Technology Libraries Inf. Guide. Delhi, Authors Press, pp: 273.

2. University of Al-Nelin, Information Technology: Teaching Book. Alkhrtum, University of Al-Nelen, pp: 5 .

3. Zash, A. Scientific research: Data and prospective. Resalat Al- Maktba, 31: 15 -26.

4. Raduan, R., 2002. Information in Arab word: Present and Prospective. Amman, Abd Al-Hamed Shuman Establishment , pp: 101-114.

5. Al Halwaji and A.A. Satar, 2002. Research and Arab Libraries between Past and Future. Cairo, pp: 271. 
6. Bu-Marifi, B., 1997. Information Technology and Communication in Modern Libraries: Appearance and its Field of Using. Amman, Dar Al-Furkan, pp: 95-98.

7. Irik, H., 1992. Computing Process of Cataloging and Classification in Library and Information Centers. Al-Reiad, Dar Al-Marikh.

8. Shraiberg,Y., 2005. Libraries and universities in the digital information environment: First steps on the way from the information society to the knowledge society. 12th Intl. Conf. Crimea, Sudak, GPNTB, http://www.gpntb.ru.

9. Al-Awaml, A.N., 1997. Methods of Scientific Research: Theoretical Fundamentals and its Practical in the Management. Amman, Jordan University, pp: 95-104.
10. Al-Hawli, A., 2002. How to Write Research Paper. Amman, Dar Al-Falah, pp: 52-56.

11. Ara'aj, S. et al., 1987. Methods of Scientific Research, Amman:Authors, pp: 67-127.

12. Khalaq, H., 1991. Methods of Cerebration, Historical Research, Aid Science and Manuscripts Realization Between Theory and Practices, Beirut, Dar Al-Nahda Al-Arabia, pp: 9-57.

13. Al-Hadi, A.M., 2000. Information and Information Technology in Start of New Century. Cairo, Al-Dar Al-Almia for Books, pp: 169-201.

14. Anonymous, 1987. Library Work: Terminal Dictionary, Moscow, Knega, pp: 21.

15. Kleb, F., 1998. Internet and its Rule in Development. Resalat Al- Maktba, 33: 41. 